\title{
Staged Implementation of an Agent Based Advanced Gas-Cooled Reactor Condition Monitoring System
}

\author{
Gordon Jahn, Stephen McArthur, Member, IEEE, Jim Reed, Dave Towle
}

\begin{abstract}
This paper introduces iMAPS, the intelligent Monitoring Assessment Panel System used by nuclear electricity generator British Energy in the UK. A Multi-Agent System design was chosen for this system and the paper describes why this technique was chosen and shows how this will allow the project to meet the short-term goals of the sponsor whilst providing a foundation for a plant-wide analysis system. Experience of building the initial stage of this system is also included, along with observations on the agent-based approach. The system was developed by the Institute for Energy and Environment at the University of Strathclyde and is in use by British Energy Generation.
\end{abstract}

Index Terms - multi-agent system, nuclear, graphite

\section{Introduction}

$\mathrm{M}$ ANY of the nuclear reactors in the UK used for electricity generation are now at a key stage in their lifetime. In the UK, the Advanced Gas-Cooled Reactors (AGRs) used for electrical power generation are made up of thousands of keyed interlocking graphite bricks that are arranged to provide around three hundred fuel channels and smaller interstitial channels used for control, instrumentation and monitoring purposes.

It is known that the internal stresses within irradiated graphite change with the total amount of radiation and an key point for the cylindrical bricks used within reactor cores is the point known as stress reversal. This is the point at which the internal stresses change from being tensile within the bore and compressive around the keyway root - the outside of the cylinder - to being tensile around the keyway root and compressive in the bore. This approximate shape of this profile is shown in figure ?? [?].

As the bricks making up the reactors in the UK approach this stress reversal point, the operator is keen to monitor the condition of the cores as closely as practically possible. In order to meet this aim, the company has instituted Monitoring Assessment Panels (MAPs) at a number of stations and given them the remit to gather and analyse information and feedback the results to station management. The long term aim of this project is to

Manuscript received December 05, 2006.

G. Jahn and S. McArthur are based at the University of Strathclyde in Glasgow, Scotland

J. Reed and D. Towle work for British Energy (Generation) Ltd in the Graphite Core Project Team

The authors would like to thank British Energy for their ongoing support of this work. This paper represents the views of the author(s) and not necessarily those of British Energy.

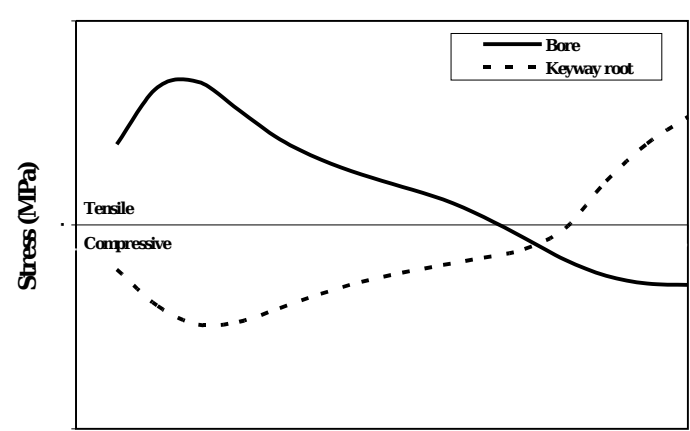

Time (full power years)

Fig. 1. Stresses within graphite moderator bricks

provide a system that can use Artificial Intelligence techniques to analysis data coming from the various system and sensors monitoring the reactor core and to help the MAP members make sense of the data. The short term aim is to provide a database to assist in the manual collection and analysis of the same data.

\section{System Design}

Condition monitoring depends on being able to infer the condition of system components from sensors and other information. Within the nuclear domain, this is particularly difficult given that reactors often run for several years between inspections and maintenance and there are relatively few sensors within the core. The primary mechanism for monitoring the core is thermocouples that can be used to monitor the temperatures and temperature gradients across the core. For this purpose, around 48 thermocouples are embedded within the graphite core itself and around 900 thermocouples are embedded within fuel stringers (at 3 thermocouples per stringer). Compounding the difficulty is that during each inspection, only a small sample of the core can be directly inspected using CCTV inspections or a channel bore monitoring unit.

Any system that was to aid with condition monitoring in this area would obviously need to look at more information than that directly available from the core itself. This data would include Fuel Grab Load Trace results where an analysis of the load on the fuelling machine hoist can give an indication of the channel shape, control rod drop 
characteristics which measure the braking speed as rods are dropped into the core and thermal-neutronic power comparisons (PANTHER) that use two separate models of the core power output to detect inadequate flow rates within the core. By also storing the operating conditions under which measurements were taken it is anticipated that models can be built to help detect any core distortion that may impact on the safety of the reactor.

The overall system design is shown in figure ??. The first stage to be implemented is the Archiving Agent and the User Interface Agent whilst the Fuel Grab Load Trace agent, the load change agent (which allows reactor conditions to be monitored), the PANTHER agent and the advanced analyses will follow as a later part of the project.

\section{System Development}

Experience of developing condition monitoring systems has proven that a flexible approach to the system is needed and this has driven recent system development including agent-based systems such as COMMAS [?] and PEDA [?] at the University of Strathclyde. As such, it was proposed that this monitoring system should be capable of gathering and analysing data from a variety of sources and making this information available in a form suitable for many different parties including managers, engineers and the Nuclear Installations Inspectorate. Furthermore, the system should be capable of performing as much analysis for these parties as possible, reducing the input on their side allowing them to focus on using the information to make decisions.

As with all new systems, an important aspect of the implementation is that it must be fully compatible with pre-existing IT infrastructures. As such, a web-based application was proposed as this would be relatively easy to deploy. It then became clear that the application based interfaces utilized in previous agent-based systems like PEDA and COMMAS with application-based interfaces were not appropriate in this case.

A new application framework was required which would allow agent behaviours to be implemented quickly and easily within the web-based interface. This framework includes a wrapper to allow JADE (the Jade Agent Development Environment) to be executed within an application server (in this case the Apache Tomcat Java Server Page (JSP)/Servlet container), a basic agent that is started for all users that $\log$ in to the system, and a number of behaviours that can be added to the agent depending on the level of access the user has to the system. Implementing new behaviours or screens for the User Interface (UI) thus requires the addition of any UI components within a JSP and the implementation of any underlying actions including communication with other agents. This simple framework maintains compatibility with agent development methodologies whilst providing the structure needed for the application.

\section{A. Archive Agent}

The first part of the system to be developed was the fact archive. This is key to storing the information about the reactor core, enabling search operations to be performed on the data and providing a robust storage mechanism. In the initial stage of development where the system could be used solely as a database, an authorized user could create facts and store these within the fact repository. As there are other research projects underway that may allow for other means of core monitoring which would have to be integrated with this system, extensibility was a key feature of this repository. As this is a fairly routine task in any condition monitoring system, a generic archive agent was written that can then be customised for each project.

As this agent was to store a large number of facts currently around 1200 hundred per reactor, per year - it would have to do so in a database in order to provide the scalability and search features that other agents would require. A very simple database schema was therefore created which contained two tables - one to store concept instances, the other to store slot values. With a little code and by leveraging the transactional and indexing capabilities of the database, a fast and highly reliable data storage engine can be added to the fact storage agent.

The schema for this database is shown in figure??.

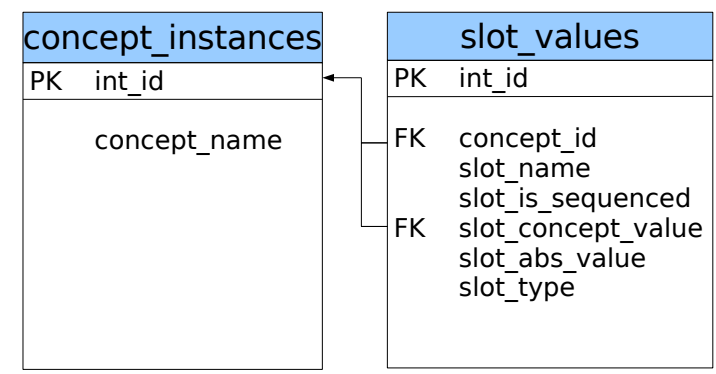

Fig. 3. Schema for fact storage agent

Storing facts is the first part of any fact storage engine - the second, more complicated, part is to allow that engine to be queried. To do this, it was decided that a bottom-up approach to the agent should be taken. In this way, as many of the FIPA performatives as possible would be set up for operation and then the application specific agents could use these at runtime to achieve their own goals. In addition to the performatives that may be sent to the archive, there is also the need to handle all applicable predicates. The difficulty here is that predicates are defined as part of the language/ontology of the system. The approach taken was therefore to keep each predicate as simple as possible and make these predicates produce a set of matching concept IDs within the database.

As can be seen from the database, each individual concept within the ontology that is stored in the database in the concept_instances table and the slots and their 


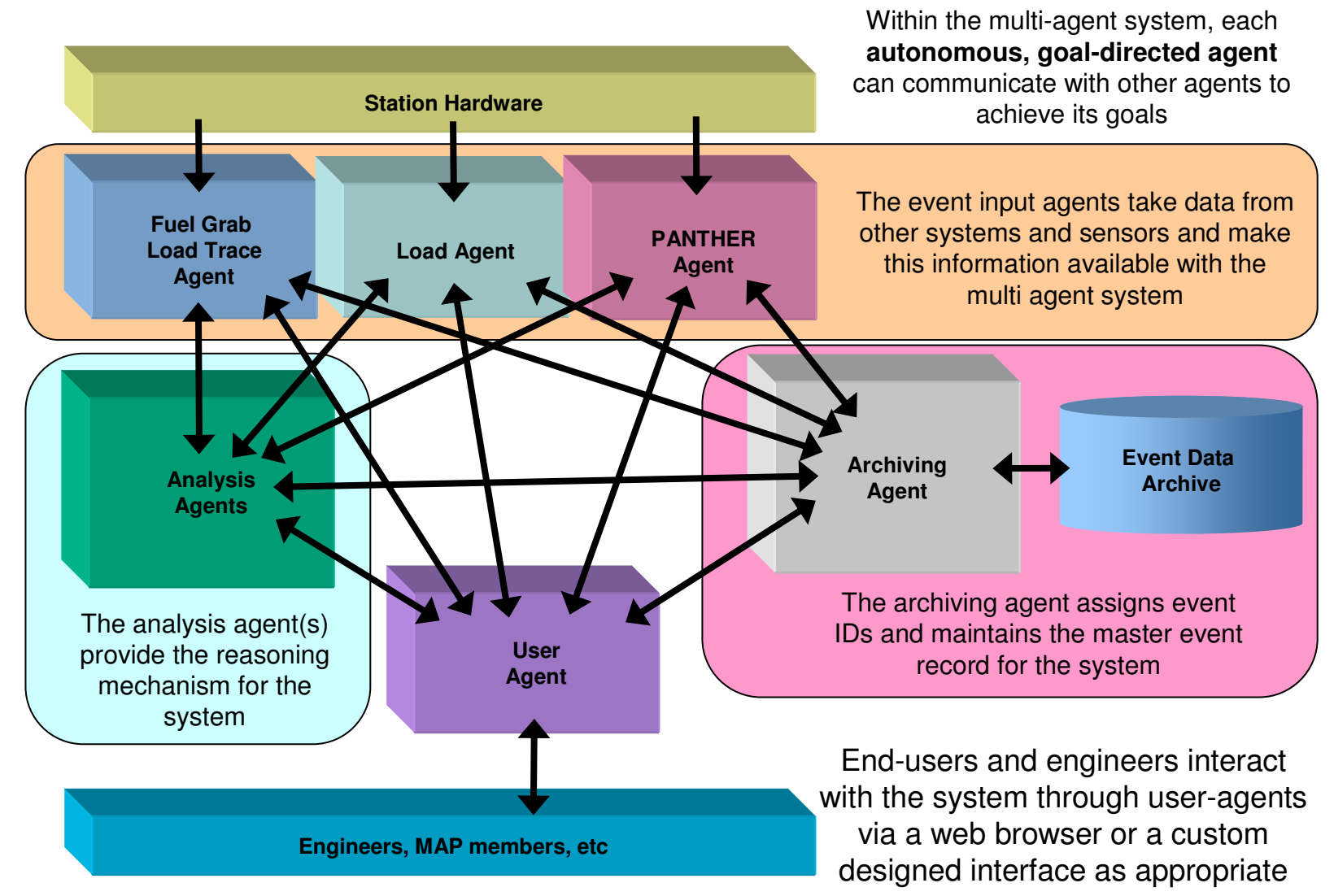

Fig. 2. Proposed British Energy Condition Monitoring System

values are stored in the slot_values table. This means that each predicate can be defined in terms on an SQL query that will return a matching result set. As an example, the occurredBefore predicate searches for ConditionMonitoringObservation facts that have a slot called startDate with a value of less than that provided in the predicate this maps directly onto an SQL query of the form:

select concept_id from slot_values where slot_name $=^{\prime}$ startDate' and slot_abs_value < '20060101T123000000';

By defining all of the predicates in this way, combinations and logical unions of these sets can be performed within SQL to return a single set of concept_ids which represents the complete result of the query sent by the agent. Codes which can automatically decompose the FIPA SL into a set of classes which can generate the complete SQL for any number of performatives have been written and have proved extremely reliable and allow the system designer to work solely with predicates and identifying referential expressions.

\section{B. User Interface Agent}

The User Interface Agent for this system has been designed in such a way that it can be used in any number of systems. This is achieved by having a very simple agent that provides management functions, such as registering and deregistering the agent, within the framework. A number of behaviours are then added to the agent as applicable to allow it to act on behalf of the user within the agent environment.

In order to aid development, an abstract behaviour implementing many of the common aspects has been coded and then extended to provide the following functionality:

- Add a new observation to the system - this creates, populates and stores the appropriate fact type within the archive

- Search the archive - this provides all the options used to create a fact as a search form allowing full searching of the archive. The search results page also provides for fact deletion and editing.

- Interactive view - this is similar to search but provides a 2 dimensional visualisation of the reactors being monitored on which observation types can be enabled or disabled, the time horizon can be modified and significance levels of observations can be toggled. The page also allows the user to add things to the this view on a temporary basis allowing them to overlay their data onto the archive without impacting on the stored data. 
- MAP Management - this view allows the Monitoring Assessment Panel representatives to specify the dates the MAPs took place on and the applicable reporting period - this information can then be used by the interactive view to provide meaningful date horizons.

\section{System Performance and Observations}

The iMAPS system presented in this paper is currently being used by British Energy and back-population of data has been carried out by British Energy staff. This was found to be a mutually beneficial process allowing system users the chance to evaluate the system and to quickly give feedback. Additionally, it was found that the list of observation types that had been made available in the initial version was completely suitable and the names and hierarchy had to be modified accordingly. The use of Protégé meant that these could be quickly modified and integrated into the system. This also bodes well for being able to add new event types into the system in the future.

Additionally, the database handling layer has been extremely robust and complex queries, in a database of around 1,000 facts, can identify the IDs of the appropriate facts in around 0.04 seconds. This performance is good considering the size of the tables and the number of facts and fact combinations being searched. Currently, the largest difficulty being faced with this system is that of data volume and retrieving the appropriate facts after identifying those that match the criteria. Due to the hierarchical structure of the facts in the database, retrieval of facts requires more than one query per fact to find all slots and slot values - this means that where you are returning 1,000 fact items to display, at least 1,000 additional database queries are required to retrieve all associated data. In this system, with the fact structure that has been employed, around 4,000 queries are required. It has been noted that if the same code was used to generate the concept ID clause within the user agent and not the archive then this data could all be retrieved with a single query and passed back to the client without the need to package the considerable amount of data into an ACL message, send it, parse it and then display it.

The idea to use the multi-agent system design and implementation in order to aid with phased deployment of this system seems to have been successful thus far. On completion of this initial phase of the project British Energy will have a fact-base ready for querying by other agents as soon as they are ready to come online. This is an important aspect of the work as the condition monitoring data can take some time to gather and store. Having this easy to query archive ready should speed development and testing of analysis systems, especially those capable of looking at channel histories.

\section{Future Work}

The next stage for this system is to fully deploy this system at power stations currently holding Monitoring
Assessment Panels. As this takes place, users will be invited to help define the next stage of the project, particularly in terms of the analyses they would like to see the system perform automatically. It is anticipated that these enhancements may see integration with other analysis systems, the development of new analysis systems including knowledge based analyses and further development of the front-end allowing users to configure more specifically how they would like to view the data or enabling the system to learn what the user is looking for in the system.

\section{References}

[1] C. Jones, "Predicting the stresses and deformations of irradiated graphite," in Proceedings of the Ageing Management of Graphite Reactor Cores 2005, Serco Assurance, 2005.

[2] S. McArthur, S. Strachan, and G. Jahn, "The design of a multiagent transformer condition monitoring system," Power Systems, IEEE Transactions on, vol. 19, no. 4, pp. 1845-1852, November 2004.

[3] E. Davidson, S. McArthur, J. McDonald, T. Cumming, and I. Watt, "Applying multi-agent system technology in practice: automated management and analysis of scada and digital fault recorder data," Power Systems, IEEE Transactions on, vol. 61, no. 2, pp. 559-567, May 2006.

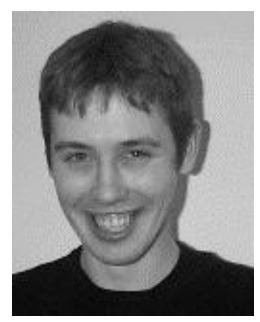

Gordon Jahn received his M.Eng (Hons) degree in 2001 from the University of Strathclyde. He currently holds the post of Research Assistant in the Institute of Energy and Environment. His research interests include, Agents, Intelligent Systems, Alarm Processing, Internet/Intranet Applications, Knowledge Engineering and AI Based Incident Identification Techniques.

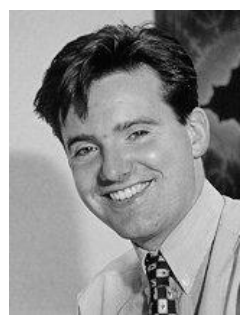

Dr Stephen McArthur is a Senior Lecturer within the Institute for Energy and Environment. He received his B.Eng. (Hons) and $\mathrm{PhD}$ degrees from the University of Strathclyde in 1992 and 1996 respectively. His research interests include Intelligent System Applications in Power Engineering, Hybrid Intelligent Systems and Intelligent Agent Technology. 